\title{
Compliance in der Händedesinfektion - 5 Jahre „AKTION Saubere Hände“
}

\author{
Thorsten Jeske $\cdot$ Christiane Reichardt $\cdot$ Petra Gastmeier
}

(C) Springer-Verlag Wien 2012

Die „AKTION Saubere Hände“ (ASH) ist eine seit dem 1. Januar 2008 durch das Bundesministerium für Gesundheit geförderte nationale Kampagne. Die Kampagne basiert auf der WHO Kampagne „Clean Care is Safer Care“ und hat die Verbesserung des Händehygieneverhaltens in deutschen Gesundheitseinrichtungen zum Ziel.

$\mathrm{Zu}$ Recht ist die nosokomiale Infektion ein hochaktuelles Thema, gilt sie doch als die häufigste Komplikation für einen Patienten während eines Krankenhausaufenthaltes.

Die Hände von Klinikpersonal stehen in der Transmissionskette bei den nosokomialen Infektionen an der ersten Stelle. Es gilt, diese Kette an den örtlichen und in den zeitlichen übertragungsrelevanten Momenten zu unterbrechen.

Mit über 1000 teilnehmenden Einrichtungen ist die ASH im internationalen Maßstab eine der größten Händehygienekampagnen der Welt und bietet:

- Strukturierte Interventionsprogramme

- Einführungskurse und Erfahrungsaustauschveranstaltungen

- Evaluierte Methoden zur Bestimmung des Händedesinfektionsmittelverbrauchs (HAND-KISS)
- Unterstützung bei der direkten Compliancebeobachtung durch die Bereitstellung von Kasuistiken und Beobachtungsbögen sowie zukünftig durch die Bereitstellung einer Beobachtungs-App

- Feedback zu Auswertungen

- Die Erstellung von Referenzdaten

Aktuelle Datenlage (Stand 2011): Es liegen Daten aus 740 Intensivstationen und 4.638 Nicht-Intensivstationen vor. 152 Krankenhäuser haben Verbrauchsdaten für die Jahre 2007, 2008, 2009 und 2010 in das HAND-KISS Modul eingegeben. Diese Einrichtungen haben eine durchschnittliche Steigerung ihres Verbrauchs um 35,9\% erreicht.

Die Zukunft der ASH: Es werden Strategien der Nachhaltigkeitsimplementierung entwickelt und umgesetzt. Die Teilnahme an der Kampagne sollte in bettenführenden Einrichtungen als anerkannter Qualitätsindikator gewertet werden. Es gilt, die Kampagne auch in Alten- und Pflegeheimen sowie ambulanten Einrichtungen noch stärker zu verbreiten.

T. Jeske $(\bowtie) \cdot$ C. Reichardt $\cdot$ P. Gastmeier Institut für Hygiene und Umweltmedizin, Charité Universitätsmedizin Berlin, Berlin, Deutschland 